\title{
Integrated Visualization of Simulation Results and Experimental Devices in Virtual-Reality Space*)
}

\author{
Hiroaki OHTANI ${ }^{1,2)}$, Akira KAGEYAMA ${ }^{3)}$, Yuichi TAMURA ${ }^{4)}$, \\ Seiji ISHIGURO ${ }^{1,2)}$ and Mamoru SHOHJI ${ }^{1)}$ \\ ${ }^{1)}$ National Institute for Fusion Science, Toki 509-5292, Japan \\ ${ }^{2)}$ The Graduate University for Advanced Studies (SOKENDAI), Toki 509-5292, Japan \\ ${ }^{3)}$ Kobe University, Kobe 657-8501, Japan \\ ${ }^{4)}$ Konan University, Kobe 658-8501, Japan
}

(Received 7 December 2010 / Accepted 31 January 2011)

\begin{abstract}
We succeeded in integrating the visualization of both simulation results and experimental device data in virtual-reality (VR) space using CAVE system. Simulation results are shown using Virtual LHD software, which can show magnetic field line, particle trajectory, and isosurface of plasma pressure of the Large Helical Device (LHD) based on data from the magnetohydrodynamics equilibrium simulation. A three-dimensional mouse, or wand, determines the initial position and pitch angle of a drift particle or the starting point of a magnetic field line, interactively in the VR space. The trajectory of a particle and the stream-line of magnetic field are calculated using the Runge-Kutta-Huta integration method on the basis of the results obtained after pointing the initial condition. The LHD vessel is objectively visualized based on CAD-data. By using these results and data, the simulated LHD plasma can be interactively drawn in the objective description of the LHD experimental vessel. Through this integrated visualization, it is possible to grasp the three-dimensional relationship of the positions between the device and plasma in the VR space, opening a new path in contribution to future research.
\end{abstract}

(C) 2011 The Japan Society of Plasma Science and Nuclear Fusion Research

Keywords: virtual reality, CAVE system, simulation data analysis, experimental device data

DOI: $10.1585 /$ pfr.6.2406027

\section{Introduction}

Virtual-reality (VR) technology is an extremely powerful and useful tool in the analysis of simulation data and the development of experimental devices, because it enables the analysis of complex structures in actual threedimensional space with a deep absorption into the VR world through scientific visualization technology. In 1997, the National Institute for Fusion Science (NIFS), Japan, installed the CompleXcope VR System based on CAVE system [1] as an instrument for scientifically analyzing simulation results. The CAVE system can produce three important visual factors stereo, immersive, and interactive views. It consists of four room-sized screens for stereo and immersive view, liquid-crystal glasses with a tracking system for stereo and interactive views, and a threedimensional mouse wand for interactive view. A viewer enters a room consisting of the four screens wearing liquidcrystal glasses through which stereo and immersive views are possible. When the viewer moves his head or walks in the room, the images on the screens are reconstructed rapidly according to his movement by using a tracking system. The viewer feels deeply immersed in the simulation model, and can view three-dimensional objects of any size

author's e-mail: ohtani.hiroaki@nifs.ac.jp

*) This article is based on the presentation at the 20th International Toki Conference (ITC20). from all directions.

NIFS has developed new software including VFIVE [2-4], AVS for CAVE, a sonification system [5], and a reactor design aid tool [6]. Through the use of these new tools, CompleXcope was adapted for scientific investigations, such as analysis of magnetohydrodynamics (MHD) simulation results for MHD dynamo [7] and spherical tokamak [8], analysis of molecular dynamics simulation results for chemical sputtering of plasma particle on a diverter [9], and analysis of particle simulation for magnetic reconnection [10].

For scientific VR visualization using the CAVE system, we developed a new approach to interactively display the integration of both simulation results and experimental device data in the VR world; a representation of simulation results in the experimental device helps us to intuitively understand the physics. In addition, this approach is useful and effective in the design and arrangement of experimental devices, because of its ability to grasp the threedimensional relationship of positions between the devices in the VR space before installing the devices in the actual vessel and to verify the state of plasma from the experimental observation port before performing the experiment.

In this paper, we introduce such a new development in scientific VR visualization at NIFS. In Sec. 2, we explain 


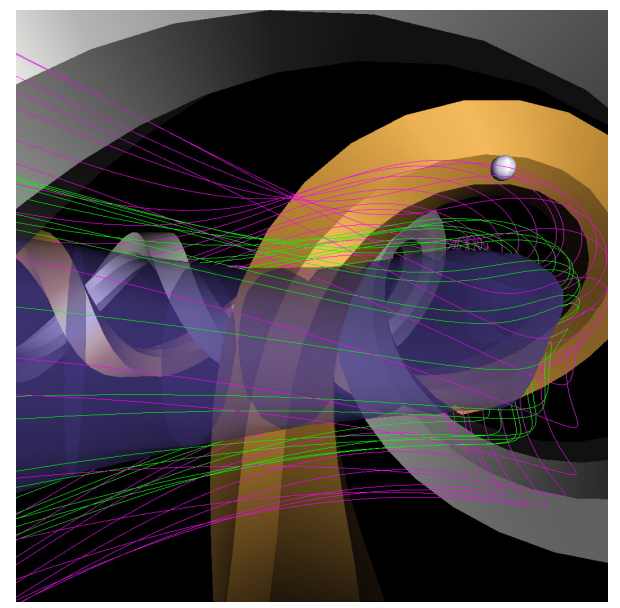

Fig. 1 Scientific visualization of the Large Helical Device (LHD) plasma by using Virtual LHD in VR space. The blue surface indicates an isosurface of plasma pressure, the white ball is a drift particle, and the green and magenta lines show the stream-line of the magnetic field and particle orbit, respectively.

the software used in the integrated VR visualization. In Sec. 3, we demonstrate that simulation results and experimental devices of the Large Helical Device (LHD) are integrated and visualized in the VR space. Finally, we summarize this paper in Sec. 4.

\section{Software}

In this section, we introduce software used in the integrated visualization of the simulation results and experimental device data in the VR space.

As shown in Fig. 1, Virtual LHD [11] calculates and visualizes in the VR space an isosurface of plasma pressure (blue surface), a stream-line of a magnetic field line (green line), and a trajectory of drift particle (magenta line). Simulation results of magnetic field, pressure, and temperature of an equilibrium plasma for input data of Virtual LHD are obtained using the MHD equilibrium simulation code HINT [12]. The initial position and the pitch angle of the drift particle are determined interactively using the threedimensional mouse wand. The wand can also designate the starting point of a magnetic field stream-line. The equation of drift motion of a particle and magnetic field line are calculated using Runge-Kutta-Huta integration method and the interpolation from the data on the grids after determining the initial point.

Virtools visualizes experimental device data, such as data of the LHD vessel device in the VR space with an objective description to create a realistic device mechanical vessel (Fig. 2) that is commercially available. Since it is difficult to visualize both the front and reverse sides of the entire polygon from the viewpoint of draw speed in real-time, only a single side of the polygon, which can be observed from inside the vacuum vessel, is described in the visualization by using Virtools. Accordingly, this drawing

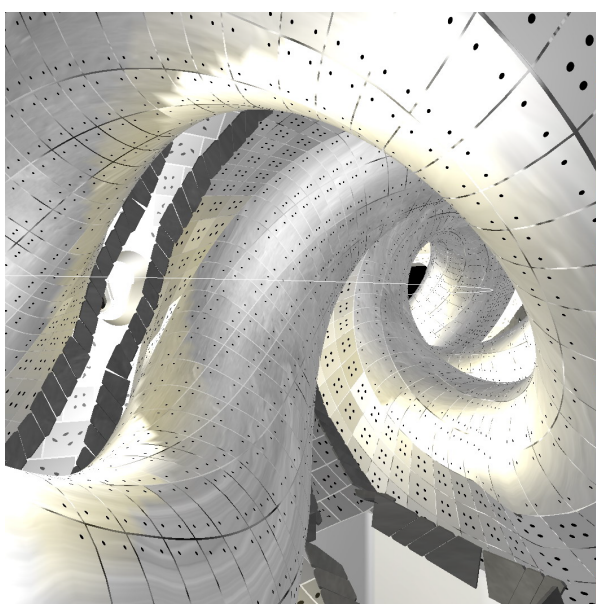

Fig. 2 Objective description of the interior of the Large Helical Device vessel in VR space.

the single side reduces the total amount of the polygon in about half. As a secondary effect of drawing only one side of objects, it is possible to observe the plasma inside the vessel even from outside the experimental device. To provide a complete objective description, the texture inside the vessel is drawn by environment mapping, and its gloss is changed by camera position. Because device data such as diverter plates and ion cyclotron heating antennae are arranged according to CAD data, in the VR space, the devices are located in the same positions as those in the actual LHD vessel.

FusionVR is also commercially available and visualizes objects by using various visualization software programs in one VR space. Viewing and integrating computer graphics (CG) created using a variety of software is difficult, and the software program that draws more realistic $\mathrm{CG}$ is generally different from the one that uses various functions to express simulation results such as isosurface, and streamline. However, FusionVR can capture OpenGL graphic data by using three-dimensional information obtained by multiple software programs and can combine them into one data set in one VR space in real-time.

When the software already contains a visualization function for virtual reality, such as the CAVE system library, the development kit FusionSDK is used. In this situation, a statement for the including FusionSDK header file, an initialization statement, an instruction statement of renderer, and a draw statement of the combined graphics must be inserted in the source file. In the present development, the helical coils in Virtual LHD (gold and silver objects in Fig. 1) are not shown because they are not required. Because the directions of the coordinate axes are different in Virtual LHD and Virtools, the axes of Virtual LHD are rotated.

\section{Visualization of Simulation Results and Device Data}

We succeeded in integrating the visualization of sim- 


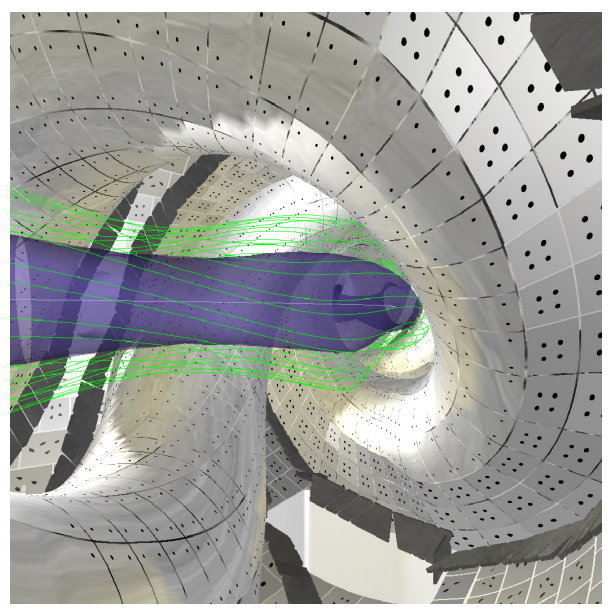

Fig. 3 Scientific VR integrated visualization of simulation results and experimental device data. The blue surface indicates an isosurface of plasma pressure, and the green line shows the stream line of magnetic field.

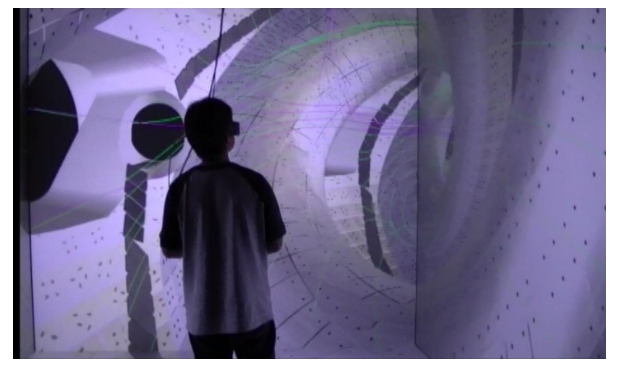

Fig. 4 Viewer in the Large Helical Device vessel in VR space and with interactive analysis of the magnetic field lines (green line) and the trajectory of drift particle (magenta line) in VR space.

ulation results obtained using Virtual LHD (using HINT code) and realistic device mechanical vessel data (the LHD vessel) obtained using Virtools based on CAD data in one VR space (Fig. 3). Integrated visualization of both realistic CG objects and objects from simulation results in one VR space is difficult to achieve. However, FusionVR and FusionSDK enable easy expression of simulation results in high-quality visualized objects by using realistic CG. As shown in Fig. 4, it is possible to walk through the vessel and interactively observe the plasma and its features, such as the magnetic field and the pressure isosurface from any perspective. Accordingly, grasping the three-dimensional relationship of the positions between the plasma and the device is easy. Since Virtual LHD function can interactively decide the initial point of the particle trajectory and the starting point of the magnetic stream-line at any point in the vessel, it is possible to determine the trajectory and the stream line to find their final points in the experimental vessel. HINT does not model the boundary regions of plasma, such as the edge and wall plasmas. If the simulation data includes the entire region in the vessel, it is possible to verify the entire the particle trajectory and magnetic field line, whose final points are on devices such as diverter

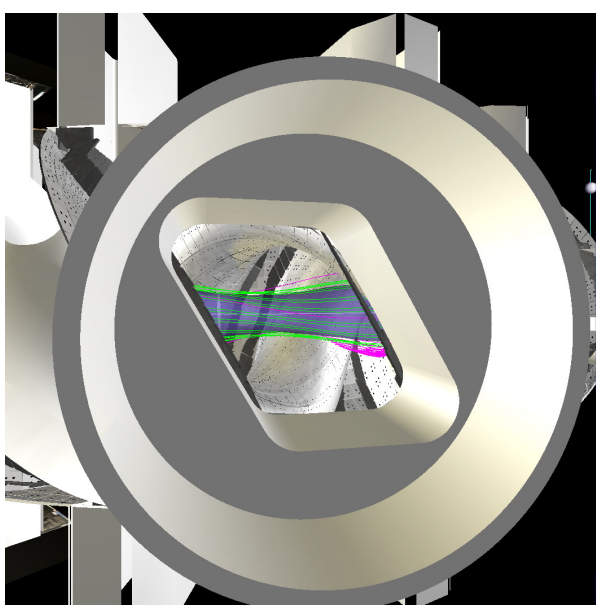

Fig. 5 View from the outer experimental observation port of the Large Helical Device. It is possible to observe the isosurface of LHD plasma colored by blue, the magnetic field line colored by green, and the particle trajectory colored by magenta.

plates in the experimental vessel.

By using this visualization in the VR space, it is possible to intuitively understand the plasma position in the virtual vessel from the port of observation. Figure 5 shows the field of vision from the outer observation port. Before setting the observation tools, it is possible to interactively observe and verify plasma in the vessel from the port as the viewer's head moves and his field of vision changes. This visualization is useful in deciding the direction of the observation device, and the position of the new device in the experimental vessel.

The representation of simulation results in the experimental device helps us to intuitively understand the physics.

From these results, we can consider several possible usages of this integrated visualization, some of which are given below. These possibilities will be the subjects of future research.

- For developing a new experimental magnetic configuration: The VR system can present a threedimensional distribution of strike points on a diverter tile in the vessel. Hence, it is easy to understand the deviation of the strike points from the diverter tile.

- For verifying the interference between the experimental equipments or between the equipment and plasma in the vessel: The distance between the equipment in the vessel and the distance or the interference between the equipment and high-energy particle/peripheral plasma can be understood three-dimensionally.

The success of this integration opens a new path in the contribution of simulation research to experiments.

\section{Summary}

In this paper, we introduced a new development in VR 
technology at NIFS. We succeeded in integrating the visualization of experimental devices and simulation results in VR space. This success indicates the possibility of intuitively understanding the physics of plasma, of aiding in the design and arrangement of the devices, and of confirming the field of vision from the observation port in VR space. We are currently developing a tool to measure the distance between two points and the angle between two planes in VR space, which will help us to grasp the distance between the plasma and the device or among the devices in the vessel. Virtual LHD reads the simulation results using HINT code. By improving the interface of input data, arbitrary simulation data can be visualized using experimental device data in VR space. VR technology is an extremely useful tool in the analysis of simulation data and the development of experimental devices. We believe that the advances introduced in this paper will enhance the study of the phenomena of plasma physics and fusion plasmas, contributing to future research.

\section{Acknowledgment}

This work was performed under the auspices of the National Institute for Fusion Science (NIFS) Collaborative Research Program (NIFS09KDAN004).
[1] C. Cruz-Neira, D.J. Sandin and T.A. DeFanti, Proc. SIGGRAPH'93, 135 (1993).

[2] A. Kageyama, Y. Tamura and T. Sato, Prog. Theor. Phys. Suppl. 138, 665 (2000).

[3] N. Ohno and A. Kageyama, Phys. Earth Planet. Inter. 163, 305 (2007).

[4] H. Ohtani, N. Ohno, N. Mizuguchi, M. Shoji and S. Ishiguro, Plasma Fusion Res. 5, 305 (2010).

[5] Y. Tamura, A. Kageyama, T. Sato, S. Fujiwara and H. Nakamura, Comput. Phys. Commun. 142, 227 (2001).

[6] N. Mizuguchi, Y. Tamura and A. Sagara, Fusion Eng. Des. 81, 2755 (2006).

[7] J. Li, T. Sato, A. Kageyama, Science 295, 1887 (2002).

[8] N. Mizuguchi, T. Hayashi and T. Sato, Phys. Plasmas 7, 940 (2000).

[9] A. Ito, Y. Wang, S. Irle, K. Morokuma and H. Nakamura, J. Nucl. Mater. 390-391, 183 (2009).

[10] H. Ohtani and R. Horiuchi, Plasma Fusion Res. 3, 054 (2008).

[11] A. Kageyama, T. Hayashi, R. Horiuchi, K. Watanabe and T. Sato, Proc. ICNSP, 138 (1998).

[12] K. Harafuji, T. Hayashi and T. Sato, J. Comput. Phys. 81, 169 (1989). 